\title{
Conformation of hexadecylphosphocholine, an anticancer drug, by molecular dynamics and NMR methods
}

\author{
Anil Saran $^{\mathrm{a}, *}$, Sudha Srivastava ${ }^{\mathrm{a}}$, Evans Coutinho $^{\mathrm{b}}$, Patrick T. T. Wong $^{\mathrm{c}}, \mathrm{H}_{\text {. Eibl }}^{\mathrm{d}}$ \\ ${ }^{2}$ Tata Institute of Fundamental Research, Homi Bhabha Road, Colaba, Bombay 400 005, India \\ ${ }^{\mathrm{b} B o m b a y}$ College of Pharmacy, Kalina, Bombay 400098 , India \\ ${ }^{c}$ Department of Biochemistry, Faculty of Medicine, University of Ottawa, Ottawa, Ont. K1H 8M5, Canada \\ ${ }^{\mathrm{a}}$ Max Planck Institute for Biophysical Chemistry, Göttingen D-3400, Germany
}

Received 3 July 1995; accepted in final form 15 January 1996

\begin{abstract}
The conformation of hexadecylphosphocholine (HPC) and its interaction with model membranes prepared from dipalmitoylphosphatidylcholine (DPPC) have been investigated by molecular dynamics (MD) and NMR methods. The results of MD studies indicate that in the global minimum energy structure, the phosphocholine head group of the drug is bent at $\tau_{18}$ and $\tau_{19}$, while the hexadecyl chain is nearly extended. In the two-dimensional nuclear Overhauser effect (NOE) experiments, intramolecular NOEs have been observed between the $N$-methyl moieties of the head group and the methylene protons of the alkyl chain. Such a folding of the molecule is seen in several of the local minimum energy structures computed by MD simulations. The interaction of HPC with DPPC as studied by ${ }^{31}$ P NMR shows a strong interaction between head groups of the two molecules.
\end{abstract}

Keywords: Anticancer drug, Hexadecylphosphocholine, Molecular dynamics, NMR spectroscopy

\section{Introduction}

Lysolecithins have a high affinity for cell membranes and cause changes in cell permeability. Besides this, they are also mediators in many cellular events [1]. Synthetic ether-lysolecithins have a profound effect on the body's immune system. Compounds with long alkyl groups $\left(\mathrm{C}_{16}\right.$ or longer) are immunostimulants, while those with short alkyl groups are immunosuppressants. Structure-activity relationship studies of ether-lysolecithins coupled with the understanding that carcinomas are devoid

\footnotetext{
* Corresponding author.
}

of ether metabolizing enzymes, led to the development of a new class of alkylphosphocholines. Hexadecylphosphocholine (HPC) (Fig. 1) is the most promising candidate in this class, with an extremely selective action against mammary carcinomas [2]. In fact, HPC has recently been marketed in Germany.

In this paper, we report the results of our investigations on the conformation of HPC by molecular dynamics (MD) and NMR methods. The drug is known to have an influence on cell membranes whose dominant component is lipids. We have thus tried to probe this aspect of its action by studying its interaction with lipid bilayers prepared from dipalmitoylphosphatidylcholine (DPPC). 


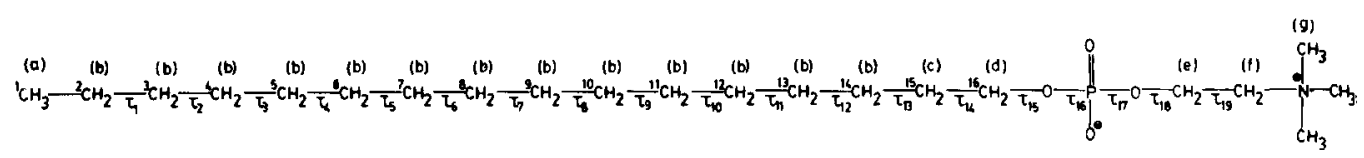

Fig. 1. Molecular structure of hexadecylphosphocholine. The torsion angles and labels for the different sets of protons have been indicated. The $\mathrm{C}_{2}-\mathrm{C}_{12}$ protons have all been labeled (b) due to their indistinguishability in the NMR spectrum.

\section{Methodology}

\subsection{Molecular dynamics}

Calculations were done on a silicon Graphics IRIS Indigo computer using software from Biosym Technologies, USA. MD simulations were run with Discover (version 2.9) and the graphical display using Insight II (version 2.3). The force field used in the computation is the Consistent Valence Force Field (CVFF) [3]. No cross-terms were used in the energy expression and the bond stretching term was represented by a simple harmonic potential. The dielectric constant has been fixed at 1.0. The following protocol was utilized to find the possible conformational states accessible to the molecule. The molecule was built in a fully extended conformation and initially minimized using 50 steps of conjugate gradients to remove any 'bad contacts' in the starting conformation. The molecule was then 'heated' up to $1500 \mathrm{~K}$ and equilibrated for sufficient time at this temperature [4]. The tempera- ture of the system at the end of the equilibration stage was $1498.7 \mathrm{~K}$. Dynamic development was then continued for a further period of 100 ps and structures from the dynamics trajectory were stored every 1 ps giving a total of 100 stored structures. Temperature control during the equilibrium stage was achieved by a direct velocity scaling, while a weak coupling to a temperature bath with a time constant of 0.1 ps was used during data collection. For each stored structure a second MD calculation at $300 \mathrm{~K}$ for 3 ps was carried out with prior equilibration. At the end of this short dynamics run, the structure was energy minimized first with 50 steps of steepest descents followed by 1000 steps of conjugate gradients or until the energy derivative fell below a threshold value of $0.001 \mathrm{kcal} \mathrm{mole}^{-1} \AA^{-1}$.

\section{2. $N M R$}

HPC was synthesized in our laboratory using the methodology reported earlier [2], while DPPC

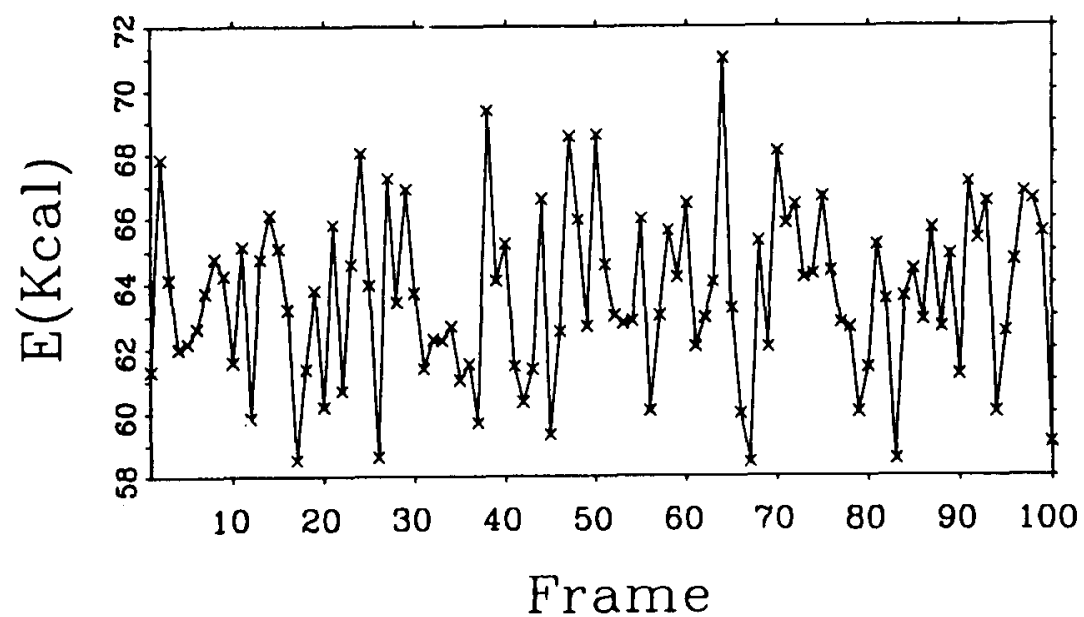

Fig. 2. Energy profile of structures sampled every 1 ps from the MD trajectory. 


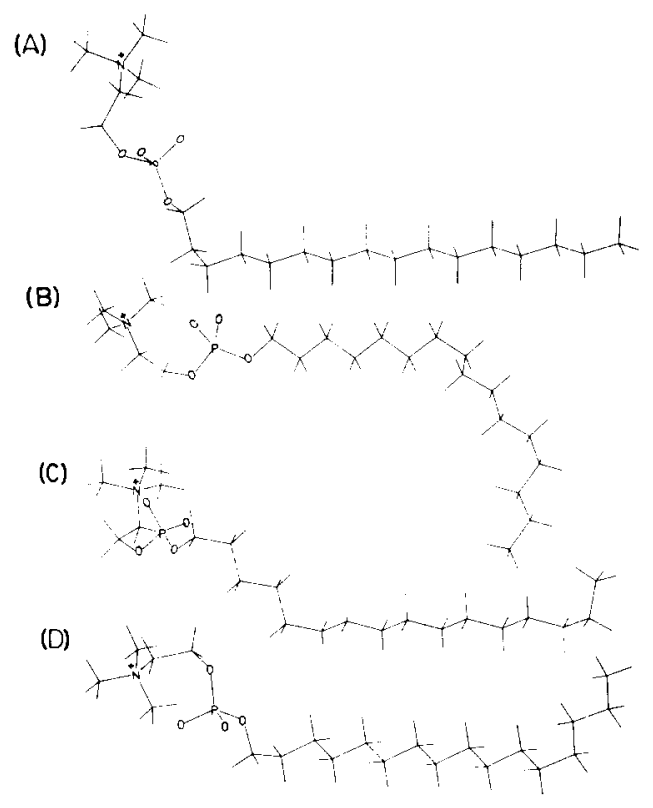

(E)

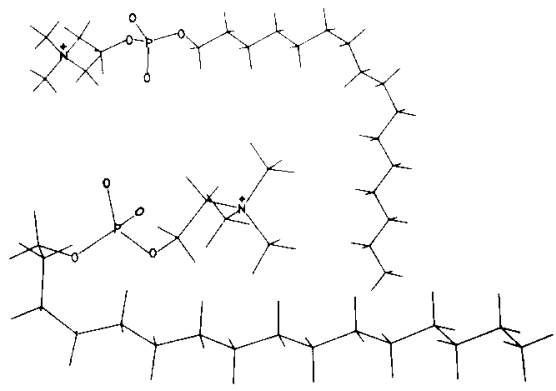

Fig. 3. Conformation of the global minimum and local minimum energy structures found by MD simulations: (A) Frame 17; (B) Frame 26; (C) Frame 45; (D) Frame 67 (global minimum); (E) Frame 83; (F) Frame 100.

was purchased from Sigma Chemical Co., USA. NMR experiments were carried out on a Bruker AMX 500 FT-NMR spectrometer. All NMR experiments were done in $\mathrm{D}_{2} \mathrm{O}$. For resonance assignments two-dimensional phase sensitive correlated spectroscopy (COSY) was carried out [5]. Two-dimensional phase sensitive nuclear Overhauser (2D-NOESY) [5] experiments were also performed to investigate the solution conformation of HPC. For both COSY and NOESY experiments the conventional pulse sequences have been used [5]. The relaxation delay $D_{1}$, in both experiments was fixed at $1 \mathrm{~s}$. The mixing time $t_{\mathrm{m}}$ in the NOESY experiments was varied from 300 to
$600 \mathrm{~ms}$. In ${ }^{31} \mathrm{P}$ NMR experiments, the protons were decoupled using the heteronuclear gated decoupling method.

Multilamellar lipid vesicles were prepared by a standard method [6]. DPPC was dissolved in chloroform and the solvent gradually evaporated by passing a stream of dry nitrogen gas into the solution. Residual solvent was removed by subjecting the dry film to vacuum for $2 \mathrm{~h}$. The drug solution in $\mathrm{D}_{2} \mathrm{O}$ was used to hydrate the lipid film. It was then heated in a water bath and vortexed to produce a uniform dispersion. Unilamellar vesicles were prepared by sonicating the dispersions using a Sonifer B-30 (Branson Sonic Power Co.) at a duty cycle of $50 \%$. The lipid to drug ratio used in these studies was $5: 1$.

\section{Results and discussion}

\subsection{Molecular dynamics}

The energy of the structures sampled every 1 ps from the MD trajectory is shown in Fig. 2. In Fig. 3 is shown the global minimum and local minimum energy structures found by MD simulation. The global minimum energy structure (Frame 67) (Fig. 3D) is characterized by $g^{+}$arrangements about $\tau_{3}, \tau_{13}, \tau_{14}$ and $\tau_{18}$ and an anticlinal form about $\tau_{19}$. The remaining torsion angles are in an extended state. Out of the 100 structures sampled from the MD trajectory, five have an energy within $1 \mathrm{kcal} \mathrm{mol}{ }^{-1}$ above the global minimum. Their conformations are depicted in Figs. 3A-3F and the associated torsion angles and energies are tabulated in Table 1. For each torsion angle $\tau_{1}$ to $\tau_{19}$, an analysis of the conformational preferences is listed in Table 2. For torsion angles in the hexadecyl chain, the extended or trans conformation always predominates (about $60 \%$ ), the remaining population being distributed between the two gauche states $g^{+}$and $g^{-}$(about $20 \%$ each). However, this does not imply that at any point in time the torsion angles in the hexadecyl chain are all extended. On analyzing the MD ensemble it is seen that, in any given structure, at least two torsion angles out of the total of fourteen in the hexadecyl chain are folded in gauche arrangements, 
Table 1

Conformation of the global minimum and local minimum energy structures found in MD simulations

\begin{tabular}{|c|c|c|c|c|c|c|}
\hline $\begin{array}{l}\text { Torsion } \\
\text { angle }\end{array}$ & $\begin{array}{l}\text { Frame } 17 \\
(0.07)^{\mathbf{a}}\end{array}$ & $\begin{array}{l}\text { Frame } 26 \\
(0.1)^{\mathrm{a}}\end{array}$ & $\begin{array}{l}\text { Frame } 45 \\
(0.8)^{\mathrm{a}}\end{array}$ & $\begin{array}{l}\text { Frame } 67 \\
\text { (Global minimum) }\end{array}$ & $\begin{array}{l}\text { Frame } 83 \\
(0.1)^{\mathbf{a}}\end{array}$ & $\begin{array}{l}\text { Frame } 100 \\
(0.6)^{\mathrm{a}}\end{array}$ \\
\hline$\tau_{1}$ & 180 & 180 & -72 & 180 & 180 & 180 \\
\hline$\tau_{2}$ & 180 & -178 & -178 & 178 & 180 & 177 \\
\hline$\tau_{3}$ & 180 & -72 & 180 & 72 & 180 & 72 \\
\hline$\tau_{4}$ & 180 & -178 & 180 & 178 & 180 & 177 \\
\hline$\tau_{5}$ & 180 & -177 & 180 & 180 & 178 & 180 \\
\hline$\tau_{6}$ & 180 & -72 & 180 & 180 & 72 & 176 \\
\hline$\tau_{7}$ & 180 & -175 & 180 & 180 & 178 & -178 \\
\hline$\tau_{8}$ & 180 & -72 & 180 & 180 & -178 & 174 \\
\hline$\tau_{9}$ & 180 & -178 & 178 & 180 & -72 & -169 \\
\hline$\tau_{10}$ & 180 & 180 & 72 & 180 & -178 & 178 \\
\hline$\tau_{11}$ & -177 & 180 & 177 & 180 & 180 & -169 \\
\hline$\tau_{12}$ & -68 & 180 & 180 & 178 & 180 & 65 \\
\hline$\tau_{13}$ & -67 & 180 & 180 & 71 & 180 & 65 \\
\hline$\tau_{14}$ & -176 & 180 & -66 & 65 & 180 & -69 \\
\hline$\tau_{15}$ & 179 & 180 & -90 & -178 & 180 & -87 \\
\hline$\tau_{16}$ & -66 & 180 & -178 & 179 & -67 & 171 \\
\hline$\tau_{17}$ & -165 & -166 & 165 & -165 & 166 & 136 \\
\hline$\tau_{18}$ & -68 & -68 & 69 & -69 & 68 & 63 \\
\hline$\tau_{19}$ & 122 & 122 & -122 & 122 & 122 & 68 \\
\hline
\end{tabular}

${ }^{a}$ Energy in $\mathrm{kcal} \mathrm{mol}^{-1}$ above the global minimum taken as energy zero.

with the remaining twelve being in an extended form. As one approaches the phosphate head group, anticlinal forms $\left(\tau \approx \pm 120^{\circ}\right)$ also begin to be populated. Thus, for $\tau_{17}$, the trans $(t)$ form is $45 \%$ populated and the anticlinal states $-120^{\circ}$ and $+120^{\circ}$ have a distribution of $29 \%$ and $26 \%$ respectively. No gauche $\left(g^{+}\right.$or $\left.g^{-}\right)$form for $\tau_{17}$ is predicted. For $\tau_{18}$ and $\tau_{19}$ the two major forms are the gauche and anticlinal states, with the trans form being disfavored. For these two torsion angles $\left(\tau_{18}\right.$ and $\tau_{19}$ ), the $g^{+}$state dominates.

\section{2. ${ }^{l} H N M R$}

\subsubsection{Resonance assignments}

In Fig. 4A is shown the ${ }^{1} \mathrm{H}$ NMR spectrum of $\mathrm{HPC}$ in $\mathrm{D}_{2} \mathrm{O}$. All protons resonate at $0-4.5 \mathrm{ppm}$. The COSY spectrum shown in Fig. 4B was used to assign the individual resonances. In the COSY spectrum, the diagonal corresponds to the onedimensional spectrum, while off-diagonal or cross-peaks establish correlations between protons that are $J$-coupled. Thus, the methyl protons (a) show a correlation to the methylene protons (b).
The resonance labeled (b) corresponds to the twelve methylene groups $\left(\mathrm{C}_{2}-\mathrm{C}_{14}\right)$ in the alkyl chain. This resonance (b) further shows a correlation to the next methylene protons (c) which are in turn coupled to neighboring methylene protons (d). The phosphocholine methylene groups (e and f) resonate downfield and show a coupling to each other. The last singlet at about $3.25 \mathrm{ppm}$ must then belong to the methyl groups ( $\mathrm{g}$ ) of the ammonium cation.

\subsubsection{Conformation}

2D-NOESY experiments can be used to establish the conformation of molecules [7]. Cross-peaks in NOESY spectra occur between protons or groups of protons that are close to each other in space $(<5 \AA)$. As mentioned earlier, several NOESY spectra were recorded with mixing times $\left(t_{\mathrm{m}}\right)$ varied from 300 to $600 \mathrm{~ms}$. Fig. 5 shows the NOESY spectrum of the drug recorded with a mixing time of $300 \mathrm{~ms}$. Two interesting NOEs are observed (circled in Fig. 5). The first occurs between the protons (g) and (b), while the second NOE is observed between the protons (d) and (b). This shows that 
Table 2

Population distribution (\%) about each torsion angle for structures sampled during MD simulation

\begin{tabular}{|c|c|c|c|c|c|}
\hline \multirow{2}{*}{$\begin{array}{l}\text { Torsion } \\
\text { angle }\end{array}$} & \multicolumn{5}{|c|}{ Conformation } \\
\hline & $g^{+}$ & $+a c$ & $t$ & $-a c$ & $g^{-}$ \\
\hline$\tau_{1}$ & 23 & & 54 & & 23 \\
\hline$\tau_{2}$ & 17 & & 70 & & 13 \\
\hline$\tau_{3}$ & 17 & & 62 & & 21 \\
\hline$\tau_{4}$ & 19 & & 68 & & 13 \\
\hline$\tau_{5}$ & 17 & & 63 & & 20 \\
\hline$\tau_{6}$ & 21 & & 59 & & 20 \\
\hline$\tau_{7}$ & 20 & & 64 & & 16 \\
\hline$\tau_{8}$ & 17 & & 65 & & 18 \\
\hline$\tau_{9}$ & 17 & & 63 & & 20 \\
\hline$\tau_{10}$ & 21 & & 63 & & 16 \\
\hline$\tau_{11}$ & 11 & & 67 & & 22 \\
\hline$\tau_{12}$ & 13 & & 66 & & 21 \\
\hline$\tau_{13}$ & 19 & & 64 & & 17 \\
\hline$\tau_{14}$ & 26 & & 47 & & 27 \\
\hline$\tau_{15}$ & 21 & 2 & 58 & 1 & 18 \\
\hline$\tau_{16}$ & 15 & & 58 & & 27 \\
\hline$\tau_{17}$ & - & 26 & 45 & 29 & - \\
\hline$\tau_{18}$ & 40 & 8 & - & 15 & 37 \\
\hline$\tau_{19}$ & 35 & 23 & - & 20 & 22 \\
\hline
\end{tabular}

The $g^{+}, t$ and $g^{-}$states refer to torsion angles in the vicinity of $60^{\circ}, 180^{\circ}$ and $-60^{\circ}$ respectively and the torsion angles $\pm 120^{\circ}$ are the anticlinal forms $(\mathrm{ac})$.

the molecule folds about the phosphate group, which brings the methyl groups of the ammonium cation in close proximity to the alkyl chain. This result is significant in view of the fact that most small molecules are highly flexible and rarely show any defined structure.

It may be mentioned here that there are a large number of low energy structures (less than $20 \mathrm{kcal} \mathrm{mol}^{-1}$ above the global minimum) in the MD simulation with short distance $(<5 \AA)$ between the sets of protons ( $\mathrm{g}$ ) and (b) and between protons (d) and (b). Specifically, in Frame 100 (Fig. 3F) (only $0.6 \mathrm{kcal} \mathrm{mol}^{-1}$ above the global minimum) a folding about $\tau_{12}$ and $\tau_{13}$ (Table 1) causes the trimethylammonium group (g) to come close to the $\mathrm{C}_{4}-\mathrm{C}_{14}$ methylene region (b) of the lipid tail. Additionally, some of the $\mathrm{C}_{12}-\mathrm{C}_{14}$ methylene groups are also close to the $\mathrm{C}_{16}$ methylene protons (d). Structure 100 is a very good model to explain the NOE's seen for HPC in solution. At least 20 out of the $100 \mathrm{MD}$ sampled structures have conformations which could explain one or both of the NOE's observed by NMR. Thus, molecular modeling strongly supports the observed NOE's.

\subsubsection{Interaction with lipid membranes}

With ${ }^{1} \mathrm{H}$ NMR it was not possible to monitor intermolecular interaction between HPC and DPPC bilayers because of the strong overlap in the regions of interest in the proton spectrum. However, the phosphorus nucleus is a convenient probe to study lipid architecture and dynamics $[8-10]$. We have therefore, tried to study this interaction by ${ }^{31} \mathrm{P}$ NMR.

Fig. 6A shows the ${ }^{31} \mathrm{P}$ NMR spectra of HPC to which varying amounts of unilamellar vesicles prepared from DPPC have been added. On addition of $10 \mu \mathrm{l}$ of DPPC to the drug (lipid to drug ratio $1: 10$ ), the ${ }^{31} \mathrm{P}$ resonance shifts by about $5.0 \mathrm{ppm}$. Further addition of DPPC does not cause substantial change in the ${ }^{31} \mathrm{P}$ chemical shift. The chemical shifts depends on the chemical environment of the nucleus and the change in the chemical shift is a result of a change in the chemical environment, which is brought about by the presence of DPPC in the vicinity of the phosphorus nucleus of HPC. This shows that the two molecules associate or interact in some way which has been probed further.

Liposomes or multilamellar vesicles prepared from DPPC have a complex ${ }^{31} \mathrm{P}$ NMR spectrum (Fig. 6B). For such systems, the fast axial rotation of the phospholipid molecules coupled with restricted motions in other directions results in a characteristic asymmetric line shape. The pattern has a very broad shoulder (around $+30 \mathrm{ppm}$ ) and a relatively sharp line at about $+16 \mathrm{ppm}$. Above the gel-to-liquid crystalline phase transition $\left(T_{\mathrm{c}}=313 \mathrm{~K}\right.$ for DPPC) a small peak at $0 \mathrm{ppm}$ can also be seen. This arises from unilamellar vesicles in coexistence with multilamellar vesicles. Below $T_{\mathrm{c}}$ where most molecular motions are 'frozen', the spectra are broadened considerably.

In Fig. 6C is shown the ${ }^{31} \mathrm{P}$ NMR spectra of liposomes containing a 1:5 mixture of HPC and DPPC. The ${ }^{31} \mathrm{P}$ resonances from the drug and DPPC are distinct and have been labeled as (a) and (b) in the figure. On increasing the temperature, the ${ }^{31} \mathrm{P}$ resonance line of the drug shifts 


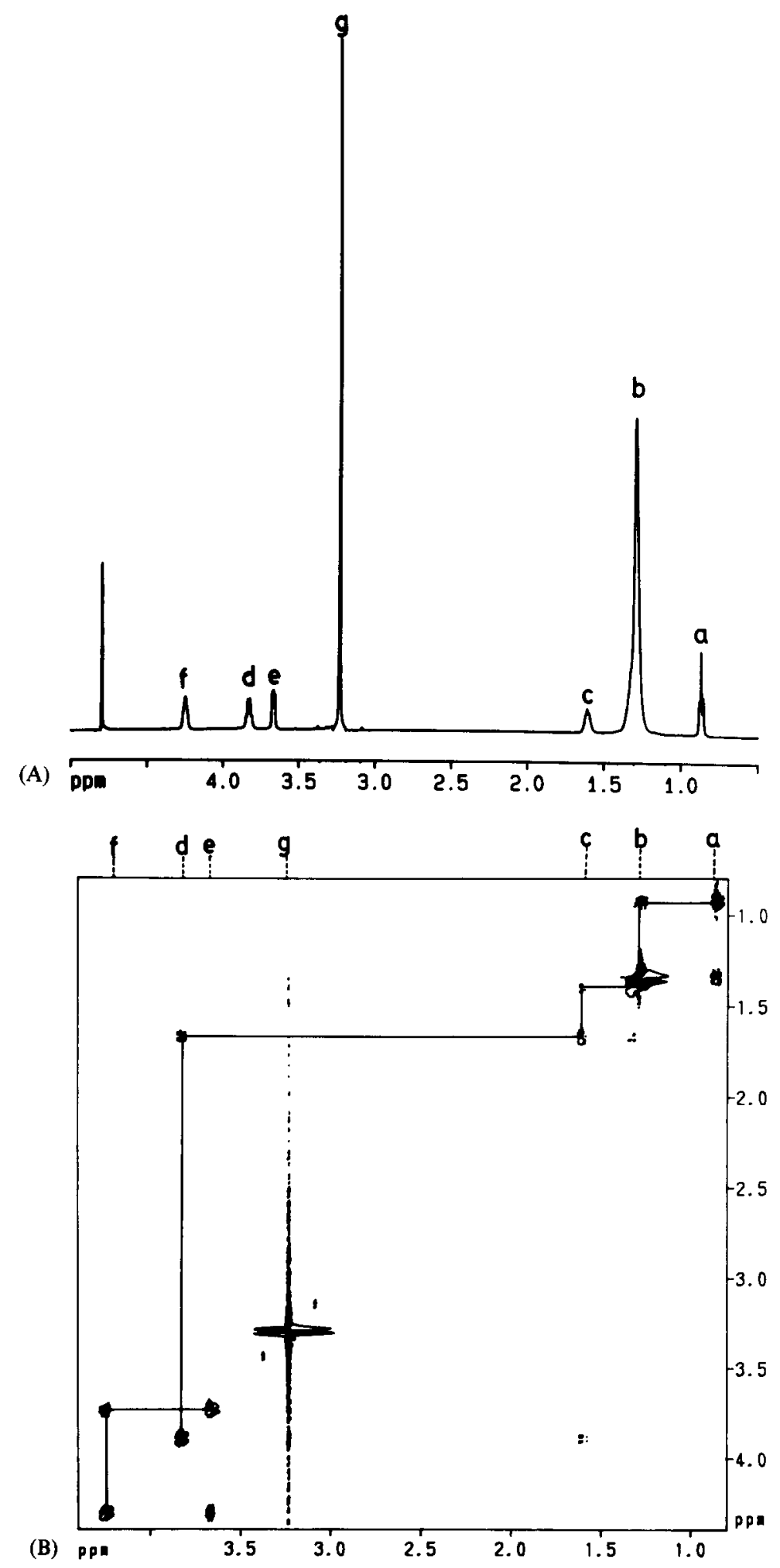

Fig. 4. (A) $500 \mathrm{MHz}{ }^{1} \mathrm{H} \mathrm{NMR}$; (B) phase sensitive COSY spectrum of $\mathrm{HPC}$ in $\mathrm{D}_{2} \mathrm{O}$ at $298 \mathrm{~K}$. 


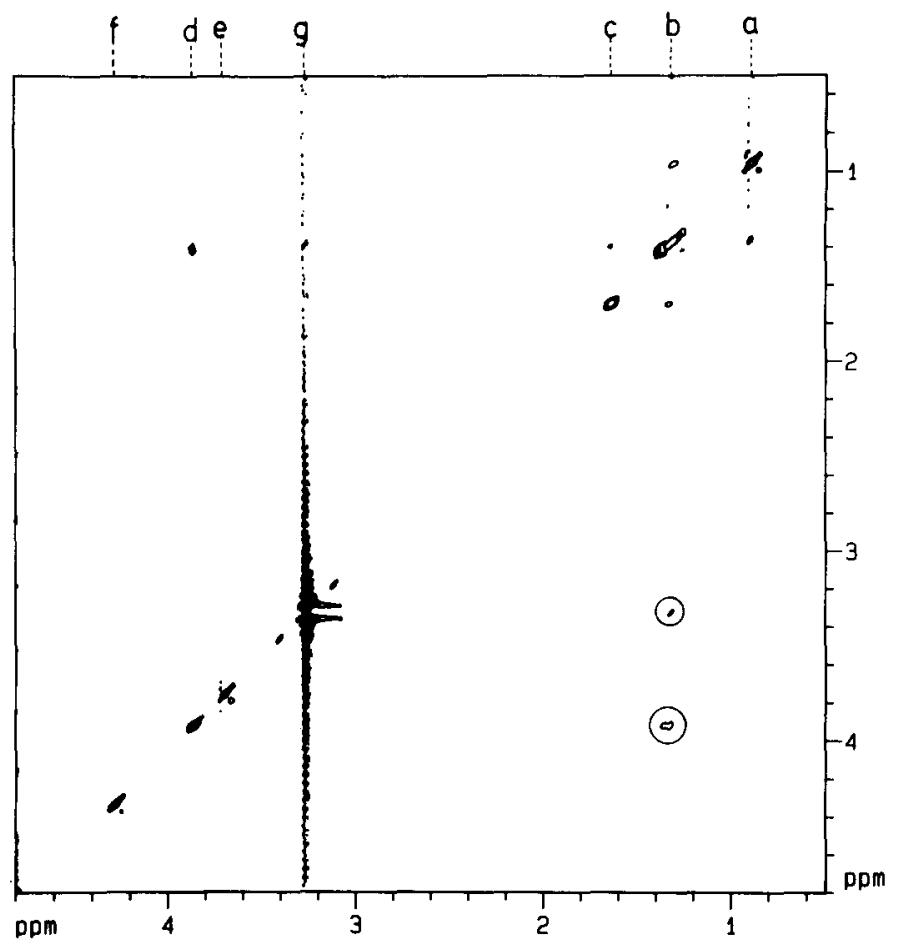

Fig. 5. Phase sensitive NOESY spectrum of $\mathrm{HPC}$ in $\mathrm{D}_{2} \mathrm{O}$ recorded with a mixing time of $300 \mathrm{~ms}$.

considerably while the ${ }^{31} \mathrm{P}$ resonance line of DPPC shifts only marginally. The largest change in the chemical shift of the ${ }^{31} \mathrm{P}$ resonance line of HPC occurs in the temperature jump from 313 to $323 \mathrm{~K}$. Also, as the temperature increases, the line width $\left(\nu_{1 / 2}\right)$ of both HPC and DPPC increases. These spectra contrast sharply with the spectra exhibited by DPPC at higher temperatures (Fig. 6B) where fast lateral motions and rapid tumbling at higher temperatures produce narrow lines. The spectra in Fig. 6C thus confirm the strong molecular interaction between HPC and DPPC which was noted earlier. Above $313 \mathrm{~K}$ (the $T_{\mathrm{c}}$ of DPPC) the melting of the gel phase and formation of the liquid crystalline phase permits a close association of the two molecules with concomitant change in vesicle size which is reflected in the changes in the chemical shifts and line widths. The results of ${ }^{31} \mathrm{P}$ NMR suggest a strong intermolecular interaction between the negatively charged phosphate group of one molecule, HPC, and the positively charged nitrogen of the second molecule, DPPC, or vice versa.

\section{Conclusions}

In conclusion, molecular dynamics studies show that for HPC the alkyl chain has a preference for an extended conformation while the phosphate head group tends to be folded in gauche arrangements. NMR studies show that the phosphate head is folded back on the alkyl chain. ${ }^{31} P$ NMR investigations indicate that HPC interacts strongly with DPPC in lipid bilayers. The most logical interaction that may be concluded is between the negatively charged phosphate group of one molecule and the positively charged trimethylammonium group of the second molecule due to ionic forces.

\section{Acknowledgments}

The facilities provided by the National Facility for High Field NMR located at TIFR and supported by DST are gratefully acknowledged. The Ministry of Human Resource and Development (MHRD), India is also thanked for the 

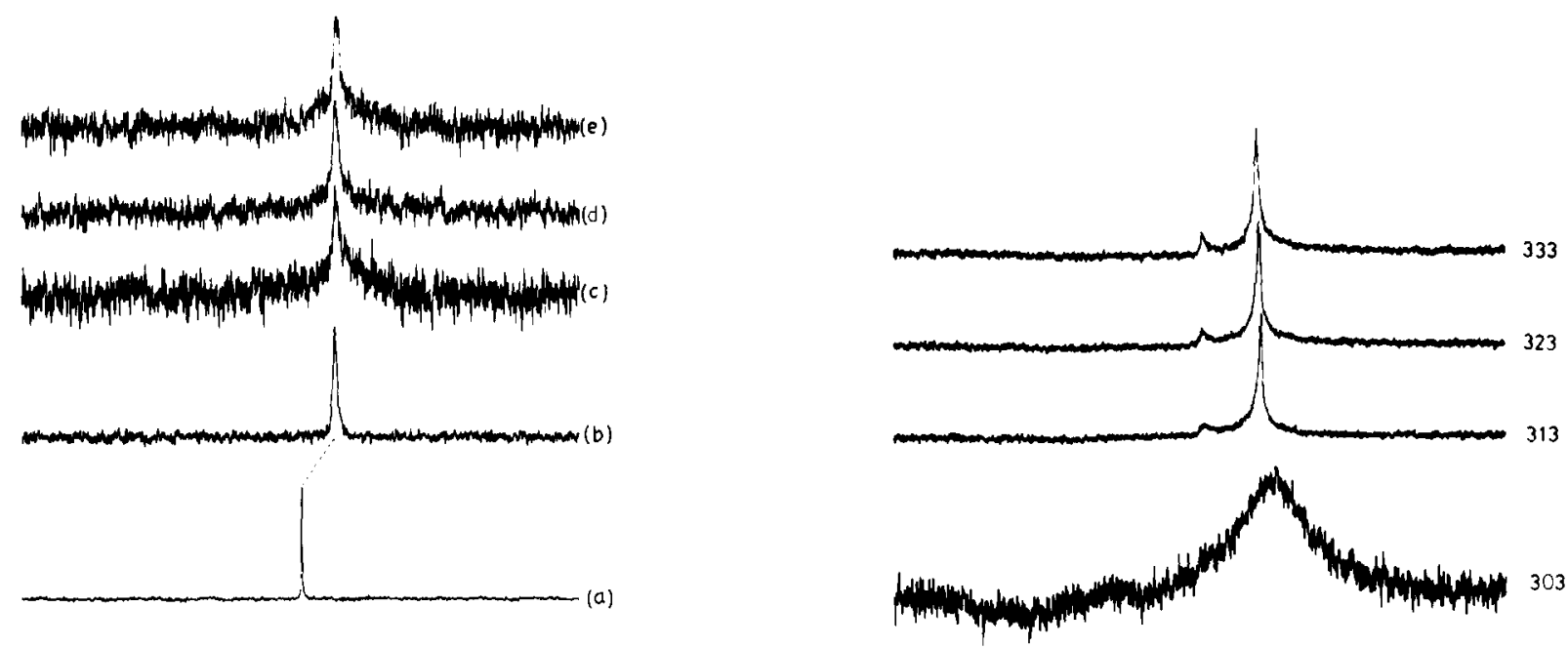

(A)

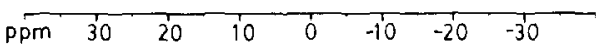

(B)
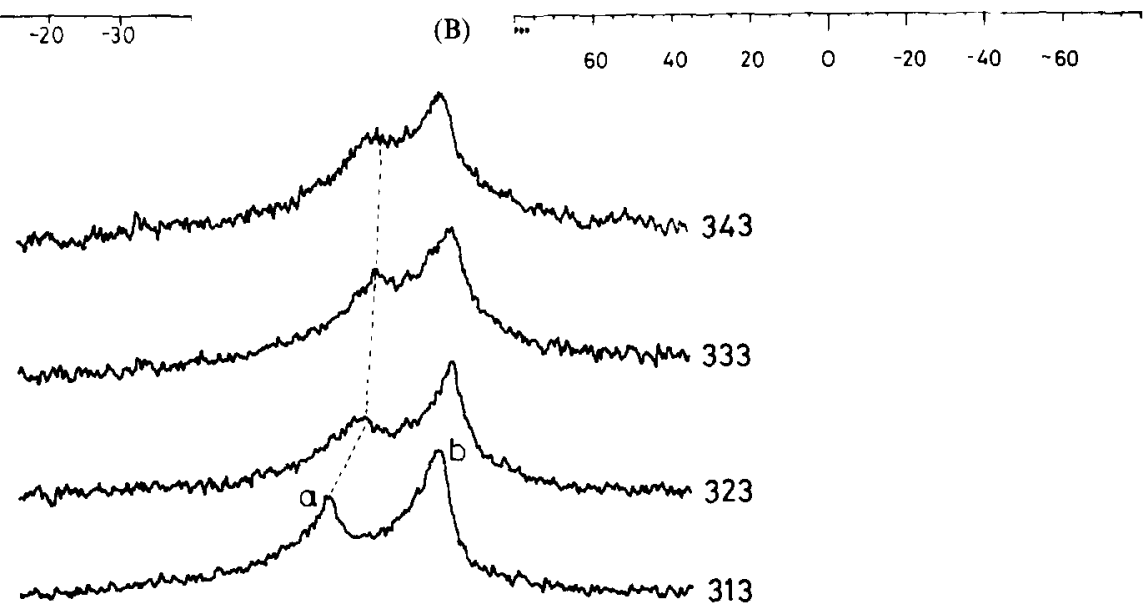

(C)

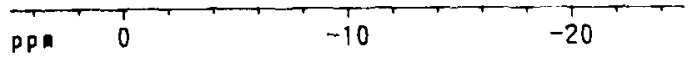

Fig. 6. (A) Proton decoupled ${ }^{31} \mathrm{P}$ NMR of (a) HPC and after addition of varying amounts of $100 \mathrm{mM} \mathrm{DPPC}(25 \mathrm{mM})(\mathrm{b}) 10 \mu \mathrm{l}$, (c) $50 \mu \mathrm{l}$ (d) $100 \mu \mathrm{l}$ and (e) $150 \mu \mathrm{l}$. The spectra have been recorded at $298 \mathrm{~K}$. (B) Proton decoupled ${ }^{31} \mathrm{P}$ NMR spectra of DPPC (100 mM) recorded at varying temperatures. A $20 \mathrm{~Hz}$ line broadening has been used. Temperatures are in $\mathrm{K}$. (C) Proton decoupled ${ }^{31} \mathrm{P}$ NMR spectra of HPC and DPPC $(1: 5)$ at varying temperatures. A $20 \mathrm{~Hz}$ line broadening has been used. Temperatures are in $\mathrm{K}$.

computational facilities at Bombay College of Pharmacy. Sudha Srivastava wishes to thank the Department of Science and Technology for support.

\section{References}

[1] F.C. Reman, R.A. Demel, J. De Gier, L.L.M. Van Deenen, H. Eibl and O. Westphal, Chem. Phys. Lipids, 3 (1969) 221.
[2] H. Eibl and C. Unger, Cancer Treatment Rev., 17 (1990) 233.

[3] P. Dauber-Osguthorpe, V.A. Roberts, D.J. Osguthorpe, J. Wolff, M. Genest and A.T. Hagler, Proteins: Struct., Function Genet., 4 (1988) 31.

[4] Discover User Guide, version 2.9.0, Biosym Technologies, San Diego, CA, January 1993.

[5] A. Bax, Two-Dimensional Nuclear Magnetic Resonance in Liquids, Delft University Press, Delft, Holland, 1984.

[6] M.W. Hill, Biochim. Biophys. Acat, 356 (1974) 117. 
[7] K. Wuthrich, NMR of Proteins and Nucleic Acids, John Wiley \& Sons, New York, 1986.

[8] D.G. Gorenstein (Ed.), Phosphorus-31 NMR, Principles and Applications, Academic Press Inc., Orlando, FL, 1984.
[9] S. Srivastava, R.S. Phadke and G. Govil, Mol. Cell. Biochem., 91 (1989) 99.

[10] J. Seelig, Biochim. Biophys. Acta, 515 (1978) 105. 